\section{O sistema de vigilância de acidentes do trabalho de Piracicaba, São Paulo, Brasil}

\author{
A monitoring system for work-related \\ accidents in Piracicaba, São Paulo, Brasil
}

\author{
Ricardo Cordeiro 1 \\ Rodolfo Andrade Gouveia Vilela 2,3 \\ Maria Angélica Tavares de Medeiros 4,5 \\ Cláudia Giglio de Oliveira Gonçalves 5 \\ Clarice Aparecida Bragantini 2 \\ Antenor J. Varolla 6,7 \\ Celso Stephan ${ }^{1}$
}

\footnotetext{
1 Faculdade de Ciências Médicas, Universidade Estadual de Campinas, Campinas, Brasil.

2 Centro de Referência de Saúde do Trabalhador, Prefeitura Municipal de Piracicaba, Piracicaba, Brasil.

3 Pós-graduação em Engenharia, Universidade Metodista de Piracicaba, Piracicaba, Brasil.

4 Faculdade de Nutrição, Pontifícia Universidade Católica de Campinas, Campinas, Brasil.

5 Faculdade de Ciências da Saúde, Universidade Metodista de Piracicaba, Piracicaba, Brasil.

6 Instituto de

Aperfeiçoamento

Tecnológico,

Piracicaba, Brasil.

7 Ministério do Trabalho

e Emprego, Brasília, Brasil.

Correspondência

R. Cordeiro

Departamento de Medicina

Preventiva e Social,

Faculdade de Ciências

Médicas, Universidade

Estadual de Campinas.

C. P. 6111, Campinas, SP

13083-970, Brasil.

cordeiro@fcm.unicamp.br
}

\section{Abstract}

The authors report on the development of a work accident monitoring system in Piracicaba, São Paulo State, Brazil, with the following characteristics: information feeding the system is obtained in real time directly from accident treatment centers; the system has universal monitoring, covering all work-related accidents in Piracicaba, regardless of the nature of the worker's employment conditions, place of work, or place of residence; health surveillance and promotion of health initiatives are triggered by identification of sentinel events; spatial distribution analysis of work-related accidents is a basic tool in designing accident awareness strategies and accident prevention policies. The system was implemented in November 2003 and by October 2004 had identified 5,320 work-related accidents, or a 3.8\% annual proportional incidence of work-related accidents in the municipal area. We illustrate spatial analysis of registered workrelated accidents and present a detailed investigation of one example of a serious accident.

Occupational Accident; Information Systems; Sentinel Surveillance; Spatial Analysis

\section{Introdução}

Os acidentes do trabalho constituem o maior agravo à saúde dos trabalhadores brasileiros 1 . Diferentemente do que o nome sugere, eles não são eventos acidentais ou fortuitos 2 , mas sim fenômenos socialmente determinados 3 , previsíveis e preveníveis. Desde 1970, quando começam os registros sistemáticos em âmbito nacional, mais de 30 milhões de acidentes foram notificados, provocando mais de $100 \mathrm{mil}$ óbitos evitáveis entre brasileiros jovens e produtivos. Apenas em 2001 foram notificados 339.645 acidentes do trabalho no Brasil 4.

Um obstáculo para o planejamento e a implementação de políticas de prevenção de acidentes do trabalho é a pequena validade destas informações, contestadas por vários autores $1,5,6,7,8,9,10$.

Um dos aspectos problemáticos das informações disponíveis é que elas se referem apenas a uma parcela dos trabalhadores do mercado formal da economia. As estatísticas oficiais brasileiras de acidentes do trabalho são elaboradas com base nas informações obtidas no documento denominado Comunicação de Acidente do Trabalho (CAT), desenvolvido pela Previdência Social com fins securitários. A emissão da CAT registra e reconhece oficialmente o acidente, estabelecendo o direito do trabalhador ao seguro acidentário junto ao Instituto Nacional de Seguridade Social (INSS). 
O preenchimento da CAT se restringe a uma parcela dos empregados "celetistas", isto é, trabalhadores com registro em carteira de trabalho regulamentado pela Consolidação das Leis do Trabalho (CLT), o que corresponde a cerca de $50,0 \%$ da força de trabalho atuando no mercado formal no Brasil. O fluxo desse documento até seu registro no INSS depende, em grande parte, de ato voluntário de sua emissão pelo empregador; do preenchimento do atestado médico contido no item II da CAT, pelo médico que atendeu o acidentado; e do seu encaminhamento à agência do INSS da área de ocorrência do acidente.

Em tais circunstâncias, associadas à quase inexistência de fiscalização, estima-se que a CAT seja emitida para, somente, cerca de $20,0 \%$ dos trabalhadores celetistas acidentados 6,7. Ainda que este documento fosse preenchido na totalidade dos casos legalmente previstos, a falta de informações sobre a ocorrência de acidentes do trabalho no setor formal da economia seria significativa. Isso porque, segundo o Plano de Benefícios da Previdência Social, a obrigatoriedade de emissão de CAT não se aplica a funcionários públicos civis e militares estatutários, trabalhadores previdenciários autônomos, empregados domésticos, garimpeiros, pescadores, proprietários, empresários, produtores rurais e religiosos.

A grande subnotificação de acidentes entre trabalhadores do mercado formal é apenas um aspecto da dificuldade em obter informações válidas sobre os acidentes do trabalho no Brasil. A ele soma-se o total desconhecimento sobre o que acontece no setor informal da economia brasileira, pela inexistência de um sistema de informação que o inclua. Este setor já abrange mais de 50,0\% da população economicamente ativa brasileira nos dias atuais (Instituto Brasileiro de Geografia e Estatística. Censo Demográfico 2000. Rio de Janeiro; 2001), estando em franco crescimento.

Pochmann 11, discutindo a desestruturação do mercado de trabalho no Brasil, demonstra que é tamanha a expansão do setor informal que, no período de 1989 a 1995, a cada 10 resultantes novos postos de trabalho, 11 foram gerados pelo segmento não organizado, enquanto 1 foi fechado no segmento organizado. Levando-se em consideração também o mercado informal da economia, estima-se que para cada 10 acidentes do trabalho ocorridos, apenas 1 é notificado no Brasil 7.

A notificação dos agravos à saúde do trabalhador é objeto de preocupação dos formula- dores de políticas de saúde desde a década de 80, como mostram iniciativas estaduais e municipais em programas e centros de referência em saúde do trabalhador. Tais experiências têm buscado responder às deficientes intervenções tanto das Delegacias Regionais do Trabalho, quanto dos serviços médicos das empresas, propondo uma ação mais global sobre a morbimortalidade relacionada ao trabalho, conjugando a assistência às ações de vigilância e contando com a participação dos trabalhadores 12 .

A vigilância à saúde do trabalhador foi incorporada ao SUS a partir da Constituição Federal de 1988, regulamentada pela Lei Orgânica da Saúde (Lei Federal n. 8.080 de 1990). A saúde do trabalhador passa, então, a ser também de responsabilidade da saúde pública, fato que confere aos programas, centros de referência em saúde do trabalhador e aos agentes sanitários do SUS, a prerrogativa da vigilância em locais de trabalho, antes tarefa exclusiva do Ministério do Trabalho, por intermédio das Delegacias Regionais.

Assim sendo, são atribuições do poder público, em seus diferentes níveis, o controle dos acidentes do trabalho, e a promoção e preservação da saúde da população trabalhadora. A formulação de políticas públicas para o enfrentamento dessa questão requer informações confiáveis sobre a distribuição, a caracterização e os determinantes dos acidentes do trabalho.

O objetivo deste texto é apresentar o sistema de vigilância de acidentes do trabalho graves e fatais que está sendo desenvolvido no Município de Piracicaba, interior do Estado de São Paulo, Brasil, buscando subsidiar a discussão sobre as políticas públicas de vigilância e prevenção destes agravos, bem como contribuir para o aprimoramento dos sistemas de informação sobre acidentes laborais no Brasil.

\section{Implantação do sistema}

O Município de Piracicaba, que em 2000 contava com 318.383 habitantes (Instituto Brasileiro de Geografia e Estatística. Censo Demográfico 2000. Rio de Janeiro; 2001), fica cerca de $150 \mathrm{~km}$ da capital de São Paulo. Posiciona-se no eixo de extensão da industrialização que vem se constituindo nas últimas duas décadas numa conturbação industrial ao longo da rodovia Anhangüera, no sentido do interior. A cidade segue características de um modelo de crescimento não sustentável, com conseqüentes ris- 
cos à saúde da população e ao meio ambiente. Piracicaba conta com um parque industrial complexo e diversificado, destacando-se empresas dos setores metalúrgico, mecânico, papel e papelão, alimentício e energético (produção de álcool) 13. A população economicamente ativa do município foi estimada, em 2003, em 138.913 trabalhadores, atuando 86.543 no mercado formal e 52.370 no mercado informal da economia (Cordeiro R. DIATEP: Diagnóstico e Controle dos Acidentes do Trabalho em Piracicaba - Relatório de Pesquisa. Campinas: Fundação de Amparo à Pesquisa do Estado de São Paulo; 2005).

Em decorrência da experiência de vigilância em acidentes do trabalho graves e fatais desenvolvida por intermédio da identificação de eventos-sentinela pelo Programa de Saúde do Trabalhador de Piracicaba, em atuação conjunta com a Subdelegacia do Ministério do Trabalho e Emprego (MTE) em Piracicaba e Sindicatos de Trabalhadores, foi proposto em 2001 um sistema de vigilância destes eventos valendo-se da notificação ocorrida nos locais de atendimento (prontos-socorros e hospitais) 13. Esta proposta foi incorporada por técnicos de diversas instituições que, em janeiro de 2002, deram início no município à pesquisa DIATEP Diagnóstico e Controle de Acidentes do Trabalho em Piracicaba, cujo objetivo geral é produzir informações e, sobretudo, tecnologia de atuação úteis ao planejamento e à execução de uma política eficiente de prevenção de acidentes do trabalho em Piracicaba. Trata-se de projeto de responsabilidade multiinstitucional, dividida entre profissionais da Universidade Estadual de Campinas (UNICAMP), da Prefeitura Municipal de Piracicaba (PMP), do MTE e da Universidade Metodista de Piracicaba (UNIMEP). O DIATEP é financiado, majoritariamente, pelo Programa de Políticas Públicas da Fundação de Amparo à Pesquisa do Estado de São Paulo (FAPESP), recebendo também recursos do Ministério da Saúde (MS), Conselho Nacional de Desenvolvimento Científico e Tecnológico (CNPq), UNICAMP, PMP, MTE, UNIMEP e UNESP.

Integra a pesquisa DIATEP a implantação do Sistema de Vigilância de Acidentes do Trabalho em Piracicaba (SIVAT-Piracicaba), o qual tem as seguintes características:

(1) As informações que alimentam o Sistema são obtidas nos locais de atendimento ao acidentado do trabalho.

(2) O Sistema tem abrangência universal dentro do município, compreendendo todos os acidentes do trabalho ocorridos em Piracicaba, independentemente da existência e da natureza do vínculo empregatício do trabalhador, de sua sede de trabalho e de seu local de moradia.
(3) As ações de vigilância e promoção à saúde são desencadeadas pela identificação de eventos sentinela 14,15 .

(4) A análise da distribuição espacial dos acidentes do trabalho, bem como dos riscos ocupacionais a eles associados, é uma ferramenta básica na elaboração de estratégias de atenção aos acidentados e de políticas de prevenção dos acidentes.

Em Piracicaba, os trabalhadores que demandam cuidados médicos decorrentes de acidentes do trabalho são atendidos em oito localidades no município: cinco pronto-socorros municipais e três hospitais privados. Após a edição do Decreto Lei Municipal n. 9.951 (Dispõe sobre a notificação obrigatória dos acidentes do trabalho através do Relatório de Atendimento ao Acidentado do Trabalho. Diário Oficial da União 2002; 12 ago), assinado em 8 de agosto de 2002 em Piracicaba, tornou-se obrigatória a notificação do atendimento a qualquer trabalhador acidentado do trabalho nestes prontosocorros e hospitais (públicos e privados), independentemente da existência ou da natureza do vínculo empregatício do trabalhador.

Em decorrência do referido Decreto Lei, a notificação passou a ser feita por intermédio do preenchimento do Relatório de Atendimento ao Acidentado do Trabalho (RAAT). Este documento contém campos para a coleta de dados de identificação do trabalhador, sua ocupação e ramo de atividade, dados da empresa para a qual trabalha (quando aplicável), local de moradia do trabalhador e de ocorrência do acidente, descrição do acidente e dados clínicos. É preenchido conjuntamente por funcionários da recepção, da enfermagem e pelo médico atendente. Após o processo de capacitação das equipes que trabalham nos serviços de atendimento de urgência da cidade (profissionais da recepção, auxiliares de enfermagem, enfermeiros e médicos), a pergunta-chave incorporada quando um acidentado dá entrada no pronto-socorro é: o que você fazia na hora do acidente? Fundamentando-se nessa questão, o acidente é caracterizado ou não como de trabalho.

Diariamente, os RAAT (http://www.pstpiracicaba.hpg.ig.com.br/leis.htm, acessado 01/Jul/ 2004) dos oito serviços de atendimento ao acidentado são coletados, suas informações digitadas em um sistema informatizado, especialmente desenhado para o armazenamento e o gerenciamento desses dados. Neste sistema já estão incorporadas as bases de informação do cadastro de ruas e código de endereçamento postal do município, da Classificação Brasileira de Ocupações (CBO) (http://www.mtecbo.gov. 
br/index.htm, acessado em 26/Abr/2005) e da Classificação Nacional de Atividades Econômicas (CNAE) (http://www.cnae.ibge.gov.br/cgibin $/$ cnae-prd.dll $/$ html $/$ Search?TIPO $=\mathrm{C} 100 \&-$ btnHie $=E$, acessado em 26/Abr/2005). A interface desse sistema com o usuário agrupa em diferentes telas, acessadas seqüencialmente, os dados das quatro partes do RAAT: Atendimento, Paciente, Acidente e Parecer Médico. A análise de consistência das informações digitadas é feita no momento da inclusão dos dados, quando vão sendo atribuídos os respectivos códigos CBO e CNAE referentes ao trabalhador acidentado, bem como as demais variáveis contidas no RAAT. Os dados referentes a trabalhadores e empresas já cadastradas no sistema ficam disponíveis para serem reaproveitados em futuras inclusões. O sistema permite a realização de diversas consultas e agrupamentos segundo tipo e gravidade do acidente, empresa, ocupação, ramo de atividade e outras características.

Com essa massa de dados, o sistema identifica acidentes graves a serem investigados, selecionados quando atendem ao menos a um dos seguintes requisitos: acidente fatal, politraumatismo, esmagamento, amputação, perfuração com perda de substância, traumatismo crânio-encefálico, fratura de coluna, lesão da medula espinal, trauma com lesão visceral, queimadura de 2 o ou 3 o grau, acidente de trabalho em menor de 16 anos.

Os acidentes selecionados pelos critérios acima são investigados por meio de inspeção nos locais das ocorrências, visando à identificação de suas causas e à execução de medidas preventivas, de modo a evitar ocorrências semelhantes.

Para tais investigações, o sistema emite um formulário com os dados do RAAT e espaços para o preenchimento das informações coletadas, que posteriormente retroalimentarão o sistema. Nessas inspeções, é preenchido um protocolo de investigação padronizado, com documentação fotográfica, e são entrevistados os acidentados (sempre que possível) e seus companheiros de trabalho, usando-se o método de investigação de Árvore de Causas 16, quando necessário. Os casos são investigados por profissionais das instituições que desenvolvem o DIATEP. Utilizam-se instrumentos legais da PMP e do MTE para a notificação e adoção de medidas preventivas. Todas as informações produzidas nas investigações são também armazenadas e gerenciadas pelo sistema informatizado, gerando relatórios periódicos.

Cada trabalhador acidentado incluído no sistema tem a ele associado quatro endereços: local de ocorrência do acidente, local (ou locais) de trabalho, local de atendimento médico e local de moradia. Para proceder à análise da distribuição espacial do risco de acidentar-se, essas informações são enviadas (juntamente com todas as demais co-variáveis associadas a cada acidentado) ao Sistema de Informação Geográfica (SIG) 17, do SIVAT-Piracicaba. Este consiste de uma base cartográfica digital georreferenciada de Piracicaba, contendo informações sobre ruas, praças e todos os cerca de 102 mil lotes urbanos da cidade. O SIG identifica automaticamente na base cartográfica, por intermédio de um procedimento técnico chamado geocoding, os locais acima referidos. As localizações podem ser visualizadas e analisadas segundo quaisquer dos atributos contidos nos RAAT. Isto permite estimar a distribuição espacial do risco de acidentes do trabalho no espaço da rua, particularmente importante para os trabalhadores informais, por meio da aplicação de técnicas de estatística espacial 18,19. Essas informações poderão ser também utilizadas no estudo das rotas mais adequadas para o resgate e transporte de trabalhadores que sofrem acidentes típicos ou de trajeto em vias públicas.

\section{Resultados}

O SIVAT-Piracicaba teve seu planejamento iniciado em 2001. Durante 2002 e a primeira metade de 2003, estabeleceram-se as parcerias necessárias e o treinamento de todas as equipes envolvidas. O sistema começou a operar em 1o de novembro de 2003 e segue em funcionamento até o presente. Os dados aqui apresentados referem-se ao período de 1o de novembro de 2003 a 31 de outubro de 2004, quando o sistema completou um ano de atividade.

Durante o período, foram atendidos nos oito pronto-socorros de Piracicaba 5.320 trabalhadores vítimas de acidentes de trabalho, sendo $4.721(88,7 \%)$ vítimas de acidentes típicos e 958 (11,3\%) vítimas de acidentes de trajeto. Os números apontam uma estimativa global de proporção anual de incidência de acidentes do trabalho que demandaram atendimento médico igual a $3,8 \%$. Dentre os trabalhadores atendidos, 156 sofreram dois acidentes, cinco sofreram três acidentes, um sofreu quatro acidentes e um sofreu cinco acidentes de trabalho no período analisado. Todos os demais sofreram um único acidente no período. Para o cálculo das estimativas abaixo-apresentadas, considerou-se sempre o primeiro acidente sofrido, para aqueles trabalhadores que se acidentaram mais de uma vez no período estudado.

Dentre os acidentados, 686 (12,9\%) eram do sexo feminino, e $4.634(87,1 \%)$ eram do sexo 
masculino, estimando-se uma proporção de incidência de acidentes de trabalho de 1,3\% e $5,5 \%$, respectivamente, para trabalhadoras e trabalhadores, diferença estatisticamente significativa $(\mathrm{a}=0,05)$.

Dentre os trabalhadores atendidos, 4.131 $(77,7 \%)$ sofreram acidentes leves, 1.041 (19,6\%) sofreram acidentes moderados e $148(2,7 \%)$ sofreram acidentes graves, dos quais $6(0,1 \%)$ faleceram durante a internação, o que aponta para uma estimativa de incidência de 4,3 acidentes do trabalho fatais para cada 100 mil trabalhadores/ano em Piracicaba. Ressalte-se que essa estimativa foi feita apenas com dados de trabalhadores que chegaram a receber atendimento médico antes do óbito e faleceram no local do atendimento, estando possivelmente subestimada. O odds masculino: feminino foi 6,4:1, 8,0:1 e 16,8:1, respectivamente, para acidentes leves, moderados e graves. Todos os acidentes fatais incidiram em trabalhadores do sexo masculino.

Dentre os trabalhadores atendidos, 2.014 $(37,9 \%)$ não necessitaram afastar-se do trabalho para tratamento, $2.986(56,1 \%)$ afastaram-se entre 1 e 15 dias, 194 (3,6\%) afastaram-se entre 16 e 30 dias e $126(2,4 \%)$ afastaram-se por mais de 30 dias em decorrência do acidente sofrido.

A Tabela 1 apresenta a distribuição dos trabalhadores acidentados segundo as causas imediatas dos acidentes sofridos, ressaltando que cerca da metade deles foi desencadeado por máquinas ou quedas de objetos.

A Tabela 2 apresenta a distribuição dos trabalhadores acidentados segundo a parte do

Tabela 1

Distribuição dos trabalhadores acidentados segundo a causa imediata do acidente. Piracicaba, São Paulo, Brasil, 2004.

\begin{tabular}{lcc}
\hline Causa imediata & $\begin{array}{c}\text { Freqüência } \\
\text { absoluta }\end{array}$ & $\begin{array}{c}\text { Freqüência } \\
\text { relativa (\%) }\end{array}$ \\
\hline Máquinas e outros equipamentos & 1.848 & 34,7 \\
Queda de objetos & 864 & 16,2 \\
Queda de altura & 810 & 15,2 \\
Veículo de transporte & 697 & 13,1 \\
Esforços/peso & 437 & 8,2 \\
Agentes físicos (calor, ruído, radiação) & 135 & 2,5 \\
Agentes químicos ou biológicos & 134 & 2,5 \\
Substâncias quentes & 104 & 2,0 \\
Animais ou plantas venenosas & 88 & 1,7 \\
Explosão/incêndio/fogo & 60 & 1,1 \\
Corrente elétrica & 17 & 0,3 \\
Outras & 126 & 2,4 \\
Total & 5.320 & 100,0 \\
\hline
\end{tabular}

corpo atingida no acidente, salientando que cerca de $2 / 3$ deles tiveram membros superiores ou inferiores atingidos.

A Tabela 3 apresenta a distribuição dos trabalhadores acidentados segundo o diagnóstico da lesão sofrida, ressaltando que cerca de $2 / 3$ deles tiveram como diagnóstico contusões ou ferimentos corto-contusos.

A Figura 1 localiza, no espaço urbano de Piracicaba, as ocorrências de acidentes do trabalho, registradas no período analisado pelos RAAT, entre os trabalhadores do mercado informal da cidade. A Figura 2 apresenta a distribuição, no espaço urbano de Piracicaba, da proporção de incidência anual de acidentes do trabalho no mercado informal da economia, obtida por um estudo caso-controle espacial 20 , no qual os casos são os acidentes mostrados na Figura 1, e os controles foram amostrados dentre trabalhadores não acidentados do mercado informal da cidade. A escala de cinza da figura varia de tons escuros para tons claros, representando o gradiente entre baixo e alto risco. As linhas dentro desta figura, que poderiam ser chamadas de "isorriscos", delimitam anéis em que a incidência estimada é igual à cota informada.

Analisando-se os RAAT preenchidos no período, foram identificados, segundo os critérios anteriormente referidos, 154 acidentes moderados ou graves, que foram investigados. Segue abaixo o relato resumido de uma das investigações feitas.

Em 1o de junho de 2004 foi atendida, em um dos pronto-socorros municipais de Piracicaba, uma diarista queixando-se de tontura, náuseas, cefaléia e falta de ar. Referia ter manuseado e inalado o produto Detax PD Plus, detergente ácido de uso profissional, usado na remoção de manchas, mofo e limo de pedras. O diagnóstico feito pelo médico plantonista foi intoxicação por ácido fluorídrico e muriático, sendo a trabalhadora tratada. No RAAT relativo a esse atendimento, foi registrado que o rótulo do produto (trazido pela trabalhadora acidentada) não alertava que o mesmo era tóxico, de uso profissional, nem sobre as precauções necessárias para seu manuseio. Tal informação, colocada dentro do Sistema, desencadeou uma visita à empresa que comercializa o produto, feita por profissionais do Centro de Referência de Saúde do Trabalhador, da Vigilância Sanitária Municipal, da DIR XV de Piracicaba e do Sindicato dos Trabalhadores na Indústria Química de Piracicaba. Na visita foram constatadas várias irregularidades: rotulagem inadequada, embalagens sem procedência, fracionamento e fabricação de produtos químicos em área física imprópria, produtos sem controle 
de qualidade, funcionários manipulando produtos químicos sem o uso de equipamentos de proteção individual (EPI), produtos químicos em prateleiras junto a gêneros alimentícios, produtos inflamáveis estocados ao lado de papelão e plástico, estocagem próxima à iluminação, corredores com passagem obstruída, fiação elétrica precária e exposta, ausência de extintores, produtos com prazo de validade vencido etc. A empresa operava sem licença da Vigilância Sanitária e sem autorização de funcionamento da Agência Nacional de Vigilância Sanitária (ANVISA). A conduta da equipe foi lavrar auto de infração, multa e fechamento do local até as irregularidades serem sanadas.

\section{Discussão}

Uma característica importante do SIVAT-Piracicaba é situar a notificação de acidentes do trabalho dentro dos serviços de urgência e emergência do SUS. Binder \& Cordeiro 6 e Cordeiro et al. 7 sugeriram, em estudos realizados em Botucatu, Estado de São Paulo, que cerca de $80,0 \%$ dos trabalhadores acidentados recebem primeiros socorros nos serviços públicos de atendimento de urgência. Estes serviços, acrescidos pelos privados, são o local estratégico escolhido para aninhar o SIVAT-Piracicaba. Esta é uma característica que diferencia este sistema do Sistema CAT, maior banco de dados sobre acidentes do trabalho no Brasil, cujo núcleo de informação situa-se no INSS.

Além de diminuir a subnotificação, alojar a geração das informações nos serviços de urgência também contribui para universalizar a abrangência do SIVAT-Piracicaba, uma vez que o SUS atende indiscriminadamente aos trabalhadores, independentemente de seu vínculo empregatício, seu local de trabalho e seu local de moradia. Assim, também aqui o SIVAT-Piracicaba diferencia-se do Sistema CAT, já que este abrange apenas uma parcela dos trabalhadores celetistas.

Com relação à atuação com base em eventos-sentinela, adotada pelo SIVAT-Piracicaba, salienta-se que esse conceito foi proposto oficialmente no Brasil por meio da Portaria $\mathrm{Mi}$ nisterial $n$. 3.120, de julho de 1998, Instrução Normativa de Vigilância em Saúde do Trabalhador do SUS, que considera intervenções nos ambientes de trabalho utilizando, entre outros, os critérios epidemiológicos obtidos dos casos mais graves e significativos.

Sistema semelhante já havia sido implantado nos Estados Unidos pelo National Institute of Occupational Safety and Health (NIOSH) ao
Tabela 2

Distribuição dos trabalhadores acidentados segundo a parte do corpo atingida durante o acidente. Piracicaba, São Paulo, Brasil, 2004.

\begin{tabular}{lcc}
\hline Parte atingida & $\begin{array}{c}\text { Freqüência } \\
\text { absoluta }\end{array}$ & $\begin{array}{c}\text { Freqüência } \\
\text { relativa (\%) }\end{array}$ \\
\hline Mãos & 1.719 & 32,3 \\
Membros inferiores (exceto pés) & 740 & 13,9 \\
Membros superiores (exceto mãos) & 704 & 13,2 \\
Olhos & 517 & 9,7 \\
Pés & 474 & 8,9 \\
Cabeça (exceto olhos) & 327 & 6,1 \\
Coluna & 254 & 4,8 \\
Tórax & 116 & 2,2 \\
Costas & 99 & 1,9 \\
Pescoço & 39 & 0,7 \\
Abdômen & 27 & 0,5 \\
Corpo todo & 17 & 0,3 \\
Outra & 287 & 5,4 \\
Total & 5.320 & 100,0 \\
\hline
\end{tabular}

Tabela 3

Distribuição dos trabalhadores acidentados segundo o diagnóstico da lesão. Piracicaba, São Paulo, Brasil, 2004.

\begin{tabular}{lcc}
\hline Diagnóstico & $\begin{array}{c}\text { Freqüência } \\
\text { absoluta }\end{array}$ & $\begin{array}{c}\text { Freqüência } \\
\text { relativa (\%) }\end{array}$ \\
\hline Contusão & 1.833 & 34,5 \\
Ferimentos corto-contusos & 1.734 & 32,6 \\
Entorse & 540 & 10,2 \\
Fratura & 405 & 7,6 \\
Queimadura & 273 & 5,1 \\
Perfuração & 100 & 1,9 \\
Politraumatismo & 82 & 1,5 \\
Amputação & 37 & 0,7 \\
Esmagamento & 36 & 0,7 \\
Intoxicação/Envenenamento & 33 & 0,6 \\
TCE & 31 & 0,6 \\
Outros & 216 & 4,1 \\
Total & 5.320 & 100,0 \\
\hline
\end{tabular}

final dos anos 80. Trata-se do Sentinel Event Notification System for Occupational Risks SENSOR 15, criado em 1987 em um projeto piloto envolvendo dez estados desse país. Consiste essencialmente do registro sistemático de determinadas ocorrências com base nos locais de atendimento (hospitais, clínicas), que notificam os casos para os serviços públicos de vi- 

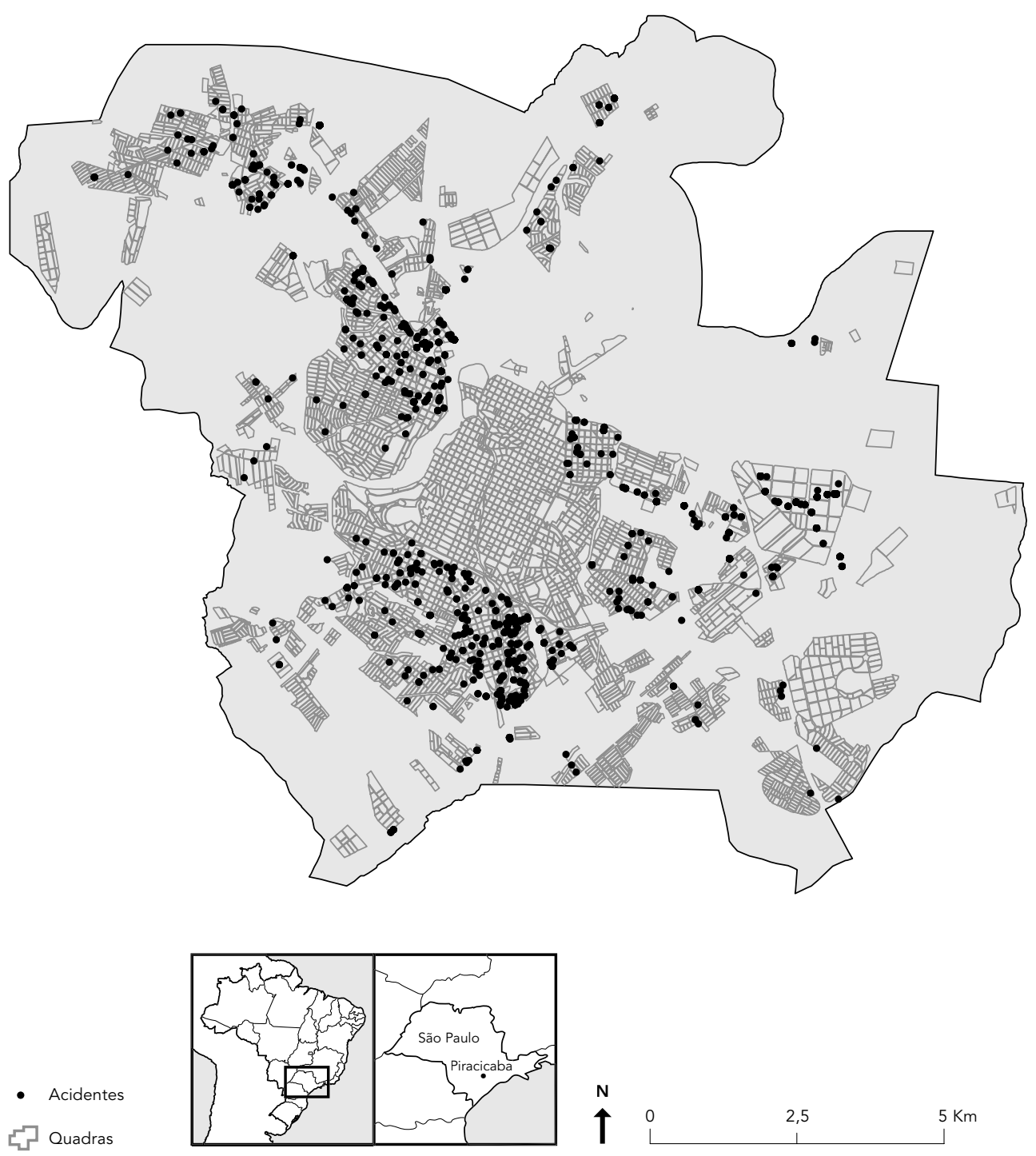

gilância. Após a análise desses registros, é desencadeada uma ação visando ao acompanhamento dos casos e intervenções nos ambientes de trabalho, de modo a prevenir a ocorrência de novos eventos. Segundo os responsáveis pelo projeto, as ações constituem o núcleo do conceito proposto pelo sistema de vigilância SENSOR.

Aliada à estratégia de tratar acidentes graves e fatais como eventos-sentinela, o SIVATPiracicaba contempla a análise de informações sobre a distribuição espacial desses acidentes, bem como dos riscos ocupacionais a eles associados. Face à necessidade de ampliação da capacidade de análise, cresce a utilização de novas técnicas no campo da epidemiologia. O espaço como categoria analítica tem trazido para a área instrumentos já consagrados em outros campos do saber (tais como a geociência, a ecologia, a meteorologia), constituindo-se em poderosas ferramentas incorporadas ao manejo e análise de dados. Embora a utilização de mapas em vigilância epidemiológica seja tradicional, a aplicação de técnicas baseadas em Siste- 
Distribuição espacial da proporção anual de incidência de acidente do trabalho no mercado informal da economia. Piracicaba, São Paulo, Brasil, 2004.

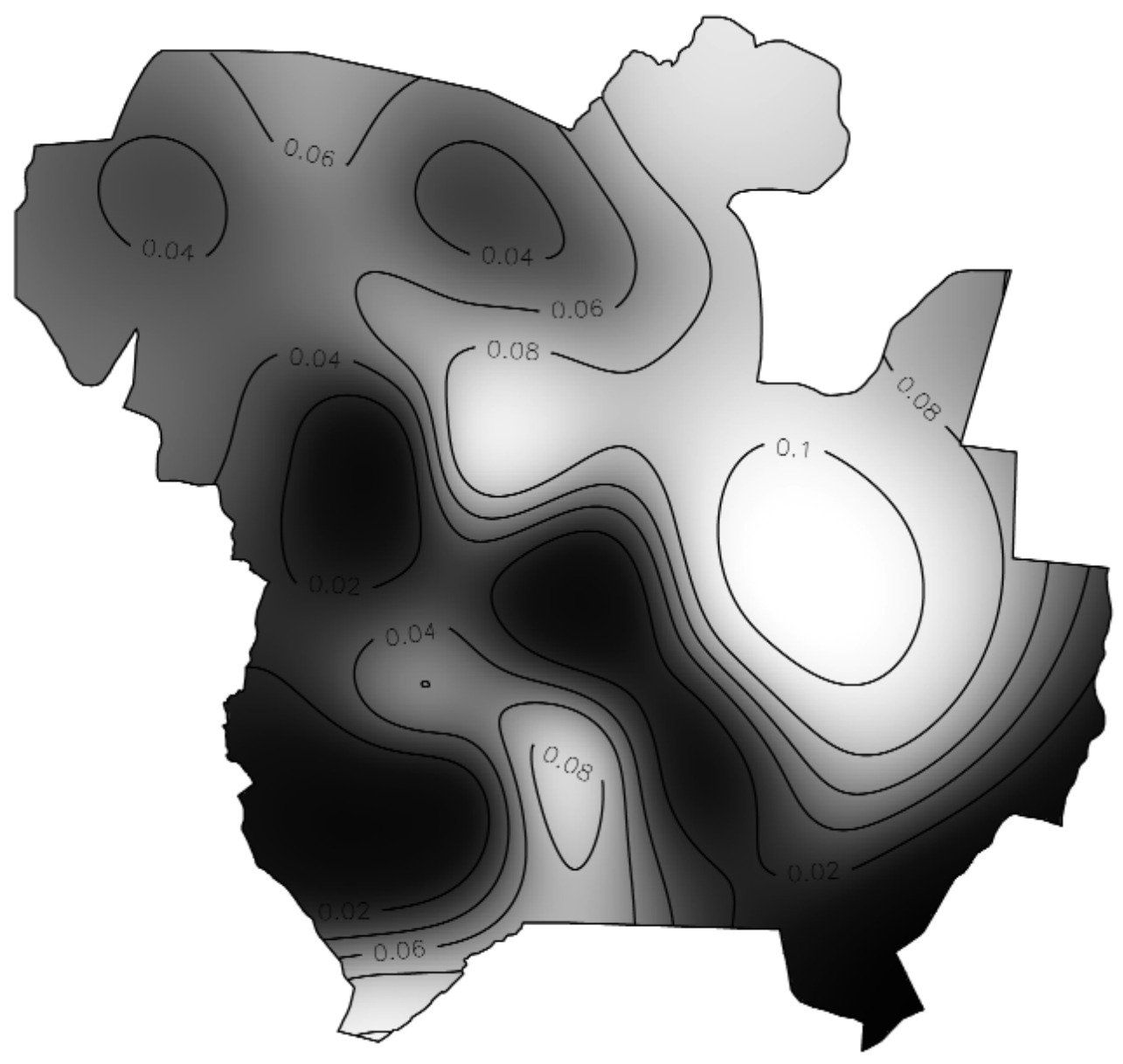

mas de Informações Georreferenciadas para a análise de distribuição espacial de acidentes do trabalho é recente. A análise de dados espaciais amplia a compreensão da relação saúde/trabalho, podendo, no âmbito dessas ações, responder questões importantes como: qual a distribuição espacial do risco de acidentes do trabalho? existe algum padrão nessa distribuição? qual é este padrão? por que ele ocorre? como alterá-lo? estabelecido esse padrão, como planejar as ações de atendimento ao acidentado?

O SIVAT-Piracicaba, em implantação, possibilita 13,21:

(1) uma melhor compreensão dos riscos existentes, por critérios epidemiológicos com informações e registros colhidos de modo sistemático;

(2) a combinação da informação vinda dos flu- xos da RAAT, das CAT e dos formulários do sistema de resgate, ampliando de modo ágil o universo para as ocorrências do mercado informal, antes praticamente ignoradas;

(3) a partir da seleção dos eventos mais significativos, o desencadeamento das ações de impacto ou ações de alcance coletivo, de modo a potencializar as medidas preventivas junto às empresas e setores econômicos, com controle social;

(4) a integração, a potencialização e amplificação das ações das diversas instituiçõoes envolvidas (Prefeitura, Ministério do Trabalho e Emprego, Universidades, Hospitais etc), com fortalecimento mútuo e melhor desempenho no exercício de suas atribuições, superando as fiscalizações fragmentadas e autuações pontuais 
ainda características predominantes da área de saúde do trabalhador;

(5) o desencadeamento de ações e negociações coletivas, com aprofundamento do conhecimento e prevenção dos riscos em determinados ramos de atividade econômica, ou por critério geográfico, quando os casos assim se apresentarem;

(6) ações coletivas estimuladas e com participação ativa do poder público, contribuindo para a construção de um ambiente favorável à prevenção, à medida que este processo participativo facilita a adesão dos diversos segmentos e órgãos de representação da sociedade civil.

O SIVAT-Piracicaba apresenta-se como uma tentativa de superação do tradicional fosso existente entre a Vigilância Sanitária e a Vigilância Epidemiológica, visto que a informação e a ação estão diretamente associadas enquanto componentes do mesmo sistema.

A despeito das potencialidades apresentadas, na implantação do SIVAT-Piracicaba alguns aspectos críticos sobressaem, como, por exemplo, as dificuldades em construir e manusear o SIG e a carência de recursos humanos para o desenvolvimento e consolidação do sistema. A maior dificuldade, entretanto, parece ser a obtenção e a manutenção da adesão ao sistema das equipes dos serviços de atendimento de urgência e emergência do município. A introdução da obrigatoriedade do preenchimento do RAAT trouxe um elemento novo à ro- tina dessas equipes, que precisam compreender a importância da construção de um sistema de informações em acidentes do trabalho para aderirem com qualidade à nova tarefa. Na fase de planejamento, investiu-se intensivamente em formação sobre as questões relativas ao SIVAT-Piracicaba para todos os profissionais envolvidos com o atendimento ao acidentado. Atualmente, a equipe que executa o DIATEP monitora continuamente a qualidade das informações contidas nos RAAT emitidos, retornando avaliações sobre a eficiência do Sistema, como estratégia para aumentar e manter a adesão dos serviços de emergência.

O SIVAT-Piracicaba é uma experiência em fase de consolidação. Estamos longe de poder avaliar se efetivamente contribui para a diminuição da incidência de acidentes do trabalho no município. Todavia, entendemos que a divulgação do desenvolvimento desse Sistema, suas características, potencialidades e entraves - ainda que em fase inicial - pode trazer alguma contribuição para o debate sobre a construção de políticas públicas de salvaguarda e promoção da saúde dos trabalhadores brasileiros. É uma possibilidade, cuja continuidade dependerá da manutenção de esforços intersetoriais, multiprofissionais, da quebra do isolamento das instituições e fundamentalmente de indivíduos que comungam com o propósito de promoção da saúde e de defesa da vida dos trabalhadores brasileiros.

\section{Resumo}

Apresenta-se a experiência de construção do Sistema de Vigilância de Acidentes do Trabalho que vem sendo desenvolvido em Piracicaba, Estado de São Paulo, que tem as seguintes características: as informações que alimentam o Sistema são obtidas diretamente, e em tempo real, nos locais de atendimento ao acidentado; o Sistema tem abrangência universal, compreendendo todos os acidentes do trabalho ocorridos em Piracicaba, independentemente da existência e da natureza do vínculo empregatício do trabalhador, de sua sede de trabalho e de seu local de moradia; as ações de vigilância e promoção à saúde são desencadeadas pela identificação de eventos sentinela; a análise da distribuição espacial dos acidentes do trabalho é uma fer- ramenta básica na elaboração de estratégias de atenção aos acidentados e de políticas de prevenção dos acidentes. O Sistema começou a operar em 10 de novembro de 2003, identificando, até 31 de outubro de 2004, 5.320 acidentados do trabalho, o que projeta uma proporção anual de incidência de acidentes de 3,8\% no município. São apresentadas ilustrações sobre a análise espacial dos acidentes registrados, bem como um resumo de uma investigação pormenorizada de um dos acidentes.

Acidente do Trabalho; Sistemas de Informação; Vigilância de Evento Sentinela; Análise Espacial 


\section{Colaboradores}

Todos os autores participaram igualmente da discussão sobre a concepção do artigo, bem como de sua redação. R. Cordeiro coordenou a elaboração do artigo, sistematizou os resultados e elaborou as Tabelas e Figuras. R. A. G. Vilela e C. Stephan aprofundaram a discussão sobre sistema de vigilância em acidentes do trabalho. M. A. T. Medeiros e C. G. O. Gonçalves aprofundaram a discussão sobre o histórico das ações de saúde do trabalhador no SUS. C. A. Bragantini e A. J. Varolla relataram a investigação que ilustra o trabalho.

\section{Agradecimentos}

Trabalho financiado pelo Programa de Políticas Públicas da Fundação de Amparo à Pesquisa do Estado de São Paulo (FAPESP 00/13719-3).

\section{Referências}

1. Hennington EA, Cordeiro R, Moreira Filho DC. Trabalho, violência e morte em Campinas. Cad Saúde Pública 2004; 20:610-7.

2. Tsai SP, Bernacki EJ, Dowd CM. The relationship between work-related and non-work-related injuries. J Community Health 1991; 16:205-12.

3. Dwyer T. Life and death at work. Industrial accidents as a case of socially produced error. New York: Plenum Press; 1991.

4. Câmara VM, Tambellini AT, Castro HA, Waissmann W. Saúde ambiental e saúde do trabalhador. Epidemiologia das relações entre a produção, o ambiente e a saúde. In: Rouquayrol MZ, Almeida Filho N, organizadores. Epidemiologia e saúde. 6a Ed. Rio de Janeiro: Medsi; 2003. p. 496-7.

5. Alves S, Luchesi G. Acidentes de trabalho e doenças ocupacionais no Brasil: a precariedade das informações. Inf Epidemiol SUS 1992; 3:7-20.

6. Binder MCP, Cordeiro R. Sub-registro de acidentes de trabalho em localidade do estado de São Paulo, 1997. Rev Saúde Pública 2003; 37:409-16.

7. Cordeiro R, Sakate M, Clemente APG, Diniz CS, Donalisio MR. Subnotificação de acidentes do trabalho não fatais em localidade do Estado de São Paulo. Rev Saúde Pública 2005; 39:254-60.

8. Carmo JC, Almeida IM, Binder MCP, Settimi MM. Acidentes do trabalho. In: Mendes R, organizador. Patologia do trabalho. Rio de Janeiro: Editora Atheneu; 1995. p. 431-55.

9. Hirata HS, Salerno MS. L'implantation d'outils statistiques sur l'organizasiotn et les conditions de travail dans les pays dits 'semi-développés - Le cas du Brésil. In: Ministère du Travail, de l’Emploi et de la Formation Professionelle, éditeur. L'usage des méthodes statistiques dans l'étude du travail. Paris: Ministère du Travail, de l'Emploi et de la Formation Professionalle; 1995. p. 117-29.

10. Possas C. Avaliação da situação atual do sistema de informação sobre doenças e acidentes do trabalho no âmbito da Previdência Social Brasileira e propostas para sua reformulação. Rev Bras Saúde Ocup 1987; 15:43-67.
11. Pochmann M. O trabalho sob fogo cruzado: exclusão, desemprego e precarização no final do século. São Paulo: Contexto; 1999.

12. Medeiros MAT. O centro de referência em saúde do trabalhador de Campinas. Trajetória de uma experiência [Dissertação de Mestrado]. Campinas: Universidade Estadual de Campinas; 2001.

13. Vilela RAG; Ricardi GVF, Iguti AM. Acidentes do trabalho graves e fatais no município de Piracicaba: desafios do Programa de Saúde do Trabalhador. Inf Epidemiol SUS 2001; 10:81-92.

14. Baker EL. Sentinel event notification system for occupational risks. Scand J Work Environ Health 1988; 14 Suppl 1:110-2.

15. Baker EL. Sentinel event notification system for occupational risks - SENSOR. The concept. Am J Public Health 1989; 79:18-21.

16. Monteau M. A practical method of investigating accident factors: principles and experimental applications. Luxembourg: Commission of the European Communities; 1977.

17. Burrough PA, McDonnell RA. Principles of geographical information systems. Oxford: Oxford Univerity Press; 1995.

18. Bailey TC, Gatrell AC. Interactive spatial data analysis. Harlow: Longman; 1995.

19. Goovaerts P. Geostatistics for natural resources evaluation. New York: Oxford Univertiy Press; 1997.

20. Bailey TC, Cordeiro R, Lourenço RW. Semi-parametric modeling of the spatial distribution of occupational accident risk in the casual labor market, Piracicaba, southeast Brazil. Scand J Environ Health; submetido.

21. Vilela RAG. Desafios da vigilância e da prevenção de acidentes do trabalho. São Paulo: Ltr Editora; 2003

Recebido em 29/Set/2004

Versão final reapresentada em 23/Mar/2005

Aprovado em 15/Abr/2005 